\title{
Blazed grating theory to minimize the non-idealities in LCoS devices
}

\author{
A. Márquez, F. J. Martínez-Guardiola, J. Francés, M. G. \\ Ramírez, E. M. Calzado, et al.
}

A. Márquez, F. J. Martínez-Guardiola, J. Francés, M. G. Ramírez, E. M. Calzado, M. Morales-Vidal, S. Gallego, I. Pascual, "Blazed grating theory to minimize the non-idealities in LCoS devices," Proc. SPIE 11136, Optics and Photonics for Information Processing XIII, 1113613 (6 September 2019); doi: $10.1117 / 12.2530290$

SPIE Event: SPIE Optical Engineering + Applications, 2019, San Diego, California, United States 


\title{
Blazed grating theory to minimize the non-idealities in LCoS devices
}

\author{
A. Márquez ${ }^{1,2, *}$, F. J. Martínez-Guardiola ${ }^{1,2}$, J. Francés ${ }^{1,2}$, M. G. Ramírez ${ }^{1,2}$, E. M. Calzado ${ }^{1,2}$, M. \\ Morales-Vidal $^{2}$, S. Gallego ${ }^{1,2}$, I. Pascual ${ }^{2,3}$ \\ ${ }^{1}$ Dept. de Física, Ing. de Sistemas y T. Señal, Universidad de Alicante, P.O. Box 99, E-03080, Alicante, Spain \\ ${ }^{2}$ I.U. Física Aplicada a las Ciencias y las Tecnologías U. de Alicante, P.O. Box 99, E-03080, Alicante, Spain \\ ${ }^{3}$ Dept. de Óptica, Farmacología y Anatomía, Univ. de Alicante, P.O. Box 99, E-03080, Alicante, Spain
}

\begin{abstract}
Parallel-aligned liquid crystal on silicon (PA-LCoS) microdisplays are widely used in spatial light modulation applications, especially in those requiring phase-only modulation. One such application area is programmable diffractive optics which plays a very important role in modern optical imaging systems or in optical interconnections for optical telecommunications. Among the multilevel diffractive optical elements (DOEs) we focus on the important case of the blazed gratings. We develop the corresponding analytical expressions for the diffracted field where, as one of the novelties in the work, an analytical expression including the fill factor and the flicker is obtained. This enables to have a model against to compare the experimental results in a number of situations where fill factor, flicker, period, and number of quantization levels are the variables. This also enables to design appropriate compensation techniques to enhance the performance of the blazed gratings.
\end{abstract}

Keywords: Liquid crystal on silicon displays, parallel aligned, phase-only modulation, spatial light modulation, flicker, fringing-field, blazed gratings, diffractive optics, Stokes polarimetry.

\section{INTRODUCTION}

Programmable optical elements displayed on spatial light modulators (SLM) play a central role in many modern photonics applications, such as in diffractive optics ${ }^{[1]}$ or reconfigurable interconnects ${ }^{[2][3]}$. Phase-only elements are usually preferable since light loss is a major concern in most of the applications. Parallel-aligned liquid-crystal on silicon (PA-LCoS) microdisplays ${ }^{[4]-[6]}$ are typically the SLM technology of choice due to their very large resolution and because they enable phase-only operation without coupled amplitude modulation.

Important efforts have been dedicated to proper modeling and characterization of PA-LCoS microdisplays ${ }^{[7][8]}$, which can be assimilated to linear variable retarders whose linear retardance varies with the applied voltage. Proper evaluation of these devices has shown that they exhibit fluctuations (flicker) in their retardance values ${ }^{[9][10]}$.

Recently ${ }^{[11]}$ we demonstrated that performance of non-uniform phase-only elements, in particular blazed gratings, can also be predicted based on the time-averaged Stokes polarimetric characterization technique ${ }^{[12]}$, which includes the existence of flicker in the retardance. Let us note that in general SLM devices exhibit a series of additional optical and electrical artifacts when images with large spatial frequency content are addressed since then cross-talk effects between neighboring pixels arise ${ }^{[13][14]}$.

In the present paper, for blazed gratings, we develop the corresponding analytical expressions for the diffracted field where, as one of the novelties in the work, an analytical expression including the fill factor and the flicker is obtained. In Section 2 we show the basic theory for far-field diffraction by blazed (or sawtooth) gratings, which are the multilevel phase elements used in the study. We consider a linear dependence of flicker amplitude with the phase level since this enables to obtain a useful analytical model. In Section 3 we provide some simulated results, showing the usefulness of our approach. Eventually the main conclusions of the paper are given in Section 4.

Optics and Photonics for Information Processing XIII, edited by Khan M. Iftekharuddin, 


\section{PIXELATED BLAZED GRATINGS: ANALYTICAL APPROACH}

When working with blazed gratings the most important parameter for their analysis is the diffraction efficiency. Usually it is expressed as the intensity diffracted to the first order normalized by the incident intensity even though other normalizations are also useful as we will show in this Section. The first order corresponds to the direction where the designer pretends to steer the incident wavefront. Next we derive the expressions for light diffracted by a multilevel pixelated blazed grating, taking into account the pixel width and the phase depth of the blazed grating. In the final step we will introduce the possibility of existence of flicker in the phase depth of the grating. We will see that we obtain a compact analytical expression. The expression enables to calculate not only the intensity diffracted to the first diffraction order but to any diffraction order. An expression taking into account all these factors does not exist in the literature and we find it useful for further analysis of the experimental and the realistic simulations which will follow in the next Sections.

We use a diffraction integral-based ${ }^{[15]}$ calculation since it provides a compact closed expression as we will show. In the following we will partly use the mathematical development presented by Chen et al. ${ }^{[16]}$, which we complete with three additional characteristics: the width of the clear aperture of the pixel, necessary to introduce the fill factor parameter, the phase flicker amplitude, and the possibility of blazed profiles with a phase-depth different of $2 \pi$ radians. We will consider pixelation only along the direction of the periodicity of the grating, so that we can use one dimensional notation.
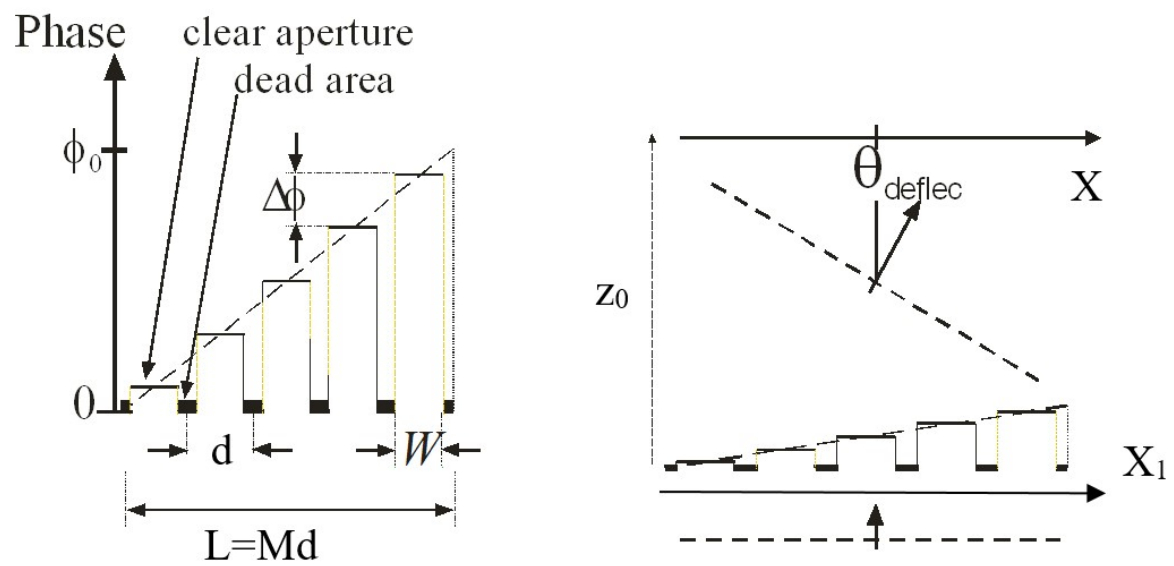

Fig. 1. Diagram of the blazed grating and the parameters involved.

In Fig. 1 we show the diagram for the multilevel pixelated blazed profile we want to model, with the following parameters: $\mathrm{d}$ is the pixel pitch, $\mathrm{W}$ is the width of the clear aperture of the pixel, $\mathrm{M}$ is the number of levels for the blazed grating, and $\mathrm{N}$ is the total number of pixels across the whole grating aperture. We note that the fill factor, a typical specification in pixelated devices, is usually defined in areal terms as $(W / d)^{2}$. According to Fig. 1, the electric field for the wavefront after traversing the grating can be considered as composed of two parts,

$$
\begin{array}{r}
a\left(x_{1}\right)=\sum_{n=0}^{(N / M)-1} \delta\left(x_{1}-n M d\right) \\
b\left(x_{1}\right)=\sum_{m=0}^{M-1} \exp (j m \Delta \phi) \operatorname{rect}\left(\frac{x_{1}-m d-W / 2}{W}\right)
\end{array}
$$

, where $a\left(x_{1}\right)$ describes de periodicity of the blazed profile, $b\left(x_{1}\right)$ the structure of one period, $\Delta \phi=\phi_{0} / M$ is the phase step for the $M$ levels composing one period, and $\varphi_{0}$ is the phase depth. And everything together we obtain, 


$$
E\left(x_{1}\right)=\left(\sum_{n=0}^{(N / M)-1} \delta\left(x_{1}-n M d\right)\right) \otimes\left(\sum_{m=0}^{M-1} \exp (j m \Delta \phi) \operatorname{rect}\left(\frac{x_{1}-m d-W / 2}{W}\right)\right)
$$

, where $\otimes$ is the convolution operation. By diffraction-integral based calculation we mean that to obtain the far-field diffraction we apply the diffraction integral, which in the case of one dimension is given by ${ }^{[15]}$,

$$
E(x)=\frac{\exp \left(j k z_{0}\right)}{j \lambda z_{0}} \exp \left(j \frac{k x^{2}}{2 z_{0}}\right)_{\infty}^{-\infty} E\left(x_{1}\right) \exp \left(-j \frac{k}{z_{0}} x x_{1}\right) d x_{1}
$$

Using the notation in the paper by Chen et al. ${ }^{[16]}$ the resultant electric field can be decomposed as $E(x)=E_{1}(x) E_{2}(x) E_{3}(x)$ where:

$$
\begin{aligned}
& E_{1}(x)= \exp \left(-j \frac{k x}{2 z_{0}}(N-M) d\right) \sin \left(\frac{k x}{2 z_{0}} N d\right) / \sin \left(\frac{k x}{2 z_{0}} M d\right) \\
& E_{2}(x)=\exp \left(-j \frac{k x}{2 z_{0}} W\right) \sin \left(\frac{k x}{2 z_{0}} W\right) / \frac{k x}{2 z_{0}} \\
& E_{3}(x)=\exp \left(-j \frac{M-1}{2}\left(-\Delta \phi+\frac{k x}{z_{0}} d\right)\right) \frac{\sin \left(\frac{M}{2}\left(-\Delta \phi+\frac{k x}{z_{0}} d\right)\right)}{\sin \left(\frac{1}{2}\left(-\Delta \phi+\frac{k x}{z_{0}} d\right)\right)}
\end{aligned}
$$

$E_{1}(x)$ is the interaction between periods; $E_{2}(x)$ is single-slit diffraction; and $E_{3}(x)$ is multislit interference in one period. To calculate the intensity, then we have, $I(x)=A_{1}(x) A_{2}(x) A_{3}(x)$, and if we make the following substitution $u=x / \lambda z_{0}$ we may rewrite the three components of the intensity expression as:

$$
\begin{gathered}
A_{1}(u)=\left|\frac{\sin (\pi u N d)}{\sin (\pi u M d)}\right|^{2} \\
A_{2}(u)=\left|\frac{\sin (\pi u W)}{\pi u}\right|^{2} \\
A_{3}(u)=\left|\frac{\sin \left(\frac{M}{2}(\Delta \phi-2 \pi u d)\right)}{\sin \left(\frac{1}{2}(\Delta \phi-2 \pi u d)\right)}\right|^{2}
\end{gathered}
$$


We note that expression $A_{2}(u)$ contains the effect of the finite extent $W$ of the clear aperture of the pixel. We substitute $\Delta \phi=\phi_{0} / M$ and put everything together:

$$
I(u)=\left|\frac{\sin (\pi u N d)}{\sin (\pi u M d)}\right|^{2}\left|\frac{\sin (\pi u W)}{\pi u}\right|^{2}\left|\frac{\sin \left(\frac{1}{2}\left(\phi_{0}-2 \pi u M d\right)\right)}{\sin \left(\frac{1}{2 M}\left(\phi_{0}-2 \pi u M d\right)\right)}\right|^{2}
$$

We note that an alternative substitution is also possible, $u=\operatorname{tg} \theta / \lambda \cong \theta / \lambda$, if we want to calculate the angular deflection $\theta$. Going back to Eqs. (6), pixelation orders occur at $u_{k}=k / d$ and for the periodicity given by the grating period $M d$ we have that the diffraction orders due to the blazed grating occur at $u_{n}=n / M d$. Therefore, pixelation orders coincide with blazed grating orders at $n=k M$.

Let us obtain the expressions at the blazed grating orders $u_{n}=n / M d$. For $A_{1}(n)$ we apply the l'Hôpital's rule since we get the indeterminate form $0 / 0$ and we obtain $A_{1}(n)=|N / M|^{2}$ independent of $n$. Then the intensity value as a function of $n$ is given by:

$$
I(n)=\left.\left(\frac{N}{M}\right)^{2}\left|\frac{\sin \left(\pi n \frac{W}{M d}\right)}{\pi n \frac{1}{M d}}\right| \frac{\sin \left(\frac{1}{2}\left(\phi_{0}-2 \pi n\right)\right)}{\sin \left(\frac{1}{2 M}\left(\phi_{0}-2 \pi n\right)\right)}\right|^{2}
$$

Let us calculate for normalization purposes the case when displaying a uniform screen $\varphi_{0}=0^{\circ}$. In the order $n=0$ we obtain:

$$
I\left(n=0 ; \phi_{0}=0\right)=\left(\frac{N}{M}\right)^{2}|W|^{2}|M|^{2}=(N W)^{2}
$$

Dividing Eq. (7) by Eq. (8), we obtain a normalized expression for the energy diffracted, as follows:

$$
I_{n o r m}(n)=\left(\frac{1}{M W}\right)^{2}\left|\frac{\sin \left(\pi n \frac{W}{M d}\right)}{\pi n \frac{1}{M d}}\right|\left|\frac{\sin \left(\frac{1}{2}\left(\phi_{0}-2 \pi n\right)\right)}{\sin \left(\frac{1}{2 M}\left(\phi_{0}-2 \pi n\right)\right)}\right|^{2}
$$

When normalized, we see that the values in Eq. (9) are independent on the extent of the grating. We note that in experiments, the diffracted intensity is usually normalized by the intensity when displaying a uniform screen, as we have just done in with Eqs. (8) and (9). Then, Eq. (9) is the expression theoretically describing most of the experiments in the literature dealing with blazed gratings ${ }^{[11]}$. We want to remark that we do not find in the literature this complete analytical expression: usually the fill factor is not considered in the derivation and only the first order is calculated. Something interesting, 
We stress the importance of Eq. (9). This is the starting point to derive the other expressions that are more specific for our purposes in the paper. Let us know model the existence of flicker. To this goal we consider that the phase depth fluctuates in time $\varphi_{0}(t)$ with the triangular profile ${ }^{[12]}$, where $\bar{\phi}_{0}$ is the average phase depth and $2 a$ is the peak-to-peak fluctuation

$$
\phi_{0}(t)=\left\{\begin{array}{cc}
\bar{\phi}_{0}-a+\frac{2 a}{T / 2} t & 0 \leq t<T / 2 \\
\bar{\phi}_{0}+3 a-\frac{2 a}{T / 2} t & T / 2 \leq t<T
\end{array}\right.
$$

Then we do the averaged calculation for the numerator and the denominator in the phase depth dependent term. From the averaging we obtain:

$$
\begin{array}{r}
\left\langle\sin \left(\frac{1}{2}\left(\phi_{0}(t)-2 \pi n\right)\right)\right\rangle=\frac{\operatorname{sen}(a / 2)}{a / 2} \operatorname{sen}\left(\frac{\bar{\phi}_{0}-2 \pi n}{2}\right) \\
\left\langle\sin \left(\frac{1}{2 M}\left(\phi_{0}(t)-2 \pi n\right)\right)\right\rangle=\frac{\operatorname{sen}(a /(2 M))}{a /(2 M)} \operatorname{sen}\left(\frac{\bar{\phi}_{0}-2 \pi n}{2 M}\right)
\end{array}
$$

Then, the averaged expression for Eq. (9) in the presence of flicker is given by:

$$
\bar{I}_{n o r m}(n)=\left(\frac{1}{M W}\right)^{2}\left|\frac{\sin \left(\pi n \frac{W}{M d}\right)}{\pi n \frac{1}{M d}}\right|\left|\frac{\frac{\operatorname{sen}(a / 2)}{a / 2} \operatorname{sen}\left(\frac{1}{2}\left(\bar{\phi}_{0}-2 \pi n\right)\right)}{\frac{\operatorname{sen}(a /(2 M))}{a /(2 M)} \operatorname{sen}\left(\frac{1}{2 M}\left(\bar{\phi}_{0}-2 \pi n\right)\right)}\right|^{2}
$$

Together with Eq. (9), this is an original result in the present paper.

\section{SIMULATED RESULTS}

Let us see some preliminary results showing the interest and potential of our analytical approach. To this goal we use Eq. (12) to check the influence of the pixel aperture, i.e. fill factor, and the flicker on the calculated intensity diffracted to the first grating order $\mathrm{n}=1$. In Fig. 2, we show these simulations as a function of the number of phase quantization levels, for no flicker (2(a)), with flicker $a=20^{\circ}(2(\mathrm{~b}))$, and with flicker $\mathrm{a}=40^{\circ}(2(\mathrm{c}))$. In each of the plots, we display the three curves produced for different values of the pixel aperture (described in the legend). The curve $\mathrm{W}=\mathrm{d}$ corresponds to the one normally used in the literature to express the diffraction efficiency of blazed gratings. Then, $\mathrm{W}=0.933 \mathrm{~d}$ corresponds to the pixel aperture of one of the commercial LCoS microdisplays PLUTO distributed by the company HOLOEYE, which is one of the most common $\mathrm{LCoS}$ devices in the literature. In the last curve, $\mathrm{W}=0.5 \mathrm{~d}$, the difference between the pixel aperture and pixel pitch is very large.

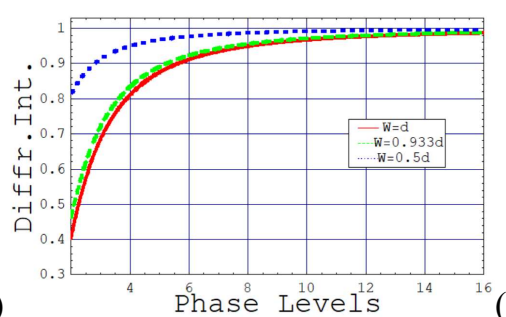

(a)

Fig. 2. Intensity diffracted to the first grating order $n=1$ as a function of the number of quantization levels of the blazed grating. We consider a phase-depth of $2 \pi$ radians. In the legend, the three values for the pixel aperture $\mathrm{W}$ considered. (a) No
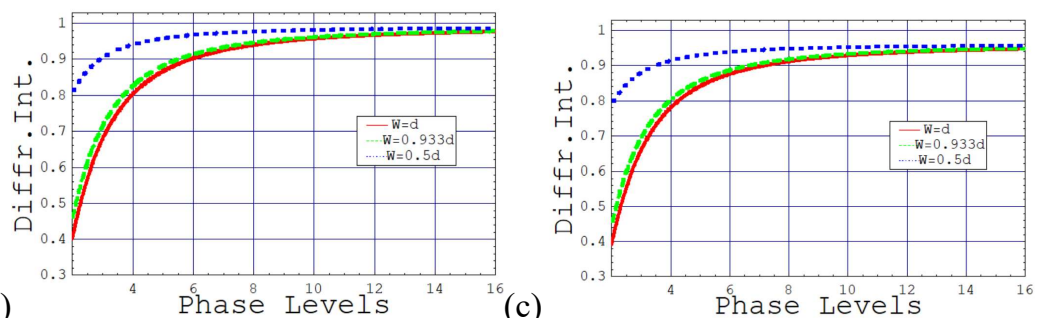

${ }^{16}(\mathrm{c})$

flicker; (b) Flicker, $\mathrm{a}=20^{\circ}$; (c) Flicker, $\mathrm{a}=40^{\circ}$. 
First thing that we see in each of the three plots is that the diffracted intensity depends on the fill factor. The difference between $\mathrm{W}=\mathrm{d}$ and $\mathrm{W}=0.933$ is noticeable at smaller number of quantization levels, and shows that it is necessary to take into account the pixel aperture to avoid errors in the calculations for the diffraction efficiency. This is not taken into account in the literature since the usual analytical expressions found in the literature ${ }^{[14]-[16]}$ implicitly consider $\mathrm{W}=\mathrm{d}$. When the pixel aperture decreases to the extreme case of $\mathrm{W}=0.5 \mathrm{~d}$, we see that the differences are very large. The next thing we analyze is the effect of flicker. As we go from plot (a) to plot (c), flicker increases and, as a result, the diffraction efficiency decreases. Change is very small between 2(a) and 2(b), and becomes more visible between 2(b) and 2(c), especially for larger number of quantization levels. However, we note that even for such a large flicker amplitude of $a=40^{\circ}$, the decrease in diffraction efficiency at large number of quantization levels is only about $5 \%$. This is consistent with the results in our previous paper ${ }^{[1]}$ where we concluded that the effect of flicker is very much application dependent. For example, in interferometry ${ }^{[10]}$ it is absolutely necessary to have minimal values of flicker. However, in programmable diffractive optics for blazed gratings, this is not a very serious degradation.

\section{CONCLUSIONS}

We have obtained analytical expression for the diffracted intensity by a multilevel blazed grating with variables fill factor, flicker, period, and number of quantization levels. These are novel expressions not existent, to the best of our knowledge, in the literature. Typical calculations in the literature consider $\mathrm{W}=\mathrm{d}$, and we have demonstrated in the simulated results that this leads to wrong values when $\mathrm{W}<\mathrm{d}$, which is always the case. Then, the pixel aperture has to be taken into account in the calculations. Eventually, we have demonstrated that flicker must be very high to impact diffraction efficiency of blazed gratings.

\section{ACKNOWLEDGEMENTS}

Work funded by Ministerio de Economía, Industria y Competitividad (Spain), projects FIS2017-82919-R (MINECO/AEI/FEDER, UE) and FIS2015-66570-P (MINECO/FEDER)); by Generalitat Valenciana (Spain), project PROMETEO II/2015/015 and GV/2019/021; and Universidad de Alicante (Spain), project GRE17-06.

\section{REFERENCES}

[1] E. Pérez-Cabré, M.S. Millán, "Liquid Crystal Spatial Light Modulator with Optimized Phase Modulation Ranges to Display Multiorder Diffractive Elements,". Appl. Sci. 9, 2592 (2019).

[2] M. Wang, L. Zong, L. Mao, A. Marquez, Y. Ye, H. Zhao, F. J. Vaquero, "LCoS SLM Study and Its Application in Wavelength Selective Switch," Photonics 4(22), 1-16 (2017).

[3] M. Salsi, C. Koebele, D. Sperti, P. Tran, H. Mardoyan, P. Brindel, S. Bigo, A. Boutin, F. Verluise, P. Sillard, M. Bigot-Astruc, L. Provost, and G. Charlet, "Mode-Division Multiplexing of $2100 \mathrm{~Gb} / \mathrm{s}$ Channels Using an LCOSBased Spatial Modulator," J. Lightwave Technol. 30, 618-623 (2012).

[4] Z. Zhang, Z. You, and D. Chu, "Fundamentals of phase-only liquid crystal on silicon (LCOS) devices," Light Sci. Appl. 3, 1-10 (2014).

[5] G. Lazarev, P.-J. Chen, J. Strauss, N. Fontaine, A. Forbes, "Beyond the display: Phase-only liquid crystal on Silicon devices and their applications in photonics," Opt. Express 27, 16206-16249 (2019).

[6] A. Márquez, A. Lizana, "Special Issue on Liquid Crystal on Silicon Devices: Modeling and Advanced Spatial Light Modulation Applications," Appl. Sci. 9, 3049 (2019).

[7] F. J. Martínez, A. Márquez, S. Gallego, J. Francés, I. Pascual, A. Beléndez, "Effective angular and wavelength modelling of parallel aligned liquid crystal devices," Opt. Lasers Eng. 74, 114-121 (2015).

[8] J. Francés, A. Márquez, F. J. Martínez-Guardiola, S. Bleda, S. Gallego, C. Neipp, I. Pascual, A. Beléndez, "Simplified physical modeling of parallel-aligned liquid crystal devices at highly non-linear tilt angle profiles," Opt. Express 26, 12723-12741 (2018). 
[9] A. Lizana, I. Moreno, A. Márquez, C. Iemmi, E. Fernández, J. Campos, and M. J. Yzuel, "Time fluctuations of the phase modulation in a liquid crystal on silicon display: characterization and effects in diffractive optics," Opt. Express 16, 16711-16722 (2008).

[10] J. García-Márquez, V. López, A. González-Vega, and E. Noé, "Flicker minimization in an LCoS spatial light Modulator," Opt. Express 20, 8431-8441 (2012).

[11]F.J. Martínez, A. Márquez, S. Gallego, M. Ortuño, J. Francés, I. Pascual, and A. Beléndez, "Predictive capability of average Stokes polarimetry for simulation of phase multilevel elements onto LCoS devices," Appl. Opt. 54, 1379-1386 (2015).

[12]F.J. Martínez, A. Márquez, S. Gallego, M. Ortuño, J. Francés, A. Beléndez, and I. Pascual, "Averaged Stokes polarimetry applied to evaluate retardance and flicker in PA-LCoS devices," Opt. Express 22, 15064-15074 (2014).

[13]B. Apter, U. Efron, and E. Bahat-Treidel, "On the fringing-field effect in liquid-crystal beam-steering devices," Appl. Opt. 43, 11-19 (2004).

[14] C. Lingel, T. Haist, and W. Osten, "Optimizing the diffraction efficiency of SLM-based holography with respect to the fringing field effect," Appl. Opt. 52, 6877-6883 (2013).

[15] J. W. Goodmann, [Introduction to Fourier-Optics, 3rd Edition], Roberts \& Company (2005).

[16] J. Chen, G. Cui, L. Kong, F. Xiao, X. Liu, and X. Zhang, "Grating lobes analysis based on blazed grating theory for liquid crystal optical-phased array,” Opt. Eng. 52(9), 97102 (2013). 